period in-vacuum undulator, high-gradient C-band linac, and a high-quality thermionic electron gun has enabled to decrease the electron beam energy, and to reduce the facility scale, for production even of hard $\mathrm{x}$-ray FEL.

The construction of SACLA started in FY2006. The first electron beam was injected in the late of February, 2011. The electron-beam acceleration to $8 \mathrm{GeV}$, as well as the observation of spontaneous $\mathrm{x}$-rays at a wavelength of 0.8 Angstrom, were achieved in March 2011. The fact that we have reached lasing after only three months of electron beam commissioning indicates (i) the validity of the basic design concept of SCSS (SPring-8 Compact SASE Source) as well as the experience accumulated during the operation of SCSS prototype machine, (ii) the certainty of the components development, design, fabrication, installation, alignment and tune-up and (iii) the adequacy of the beam commissioning strategy, meaning that the project has moved ahead with extraordinaire smoothness.

We are continuing the commissioning of SACLA in order to deliver higher intensity and shorter wavelength X-ray laser with higher stability. SACLA will be open for public users in March, 2012.

\section{$1 \mathrm{SI}-02$}

\section{非結晶生体粒子のに対する低温コヒーレントX線回折顯微鏡法実験}

Coherent X-ray diffraction microscopy of non-crystalline biological particles at cryogenic temperatures

Masayoshi Nakasako ${ }^{1,2}$, Masaki Yamamoto ${ }^{2}\left({ }^{1}\right.$ Department of Physics, Keio University, ${ }^{2}$ RIKEN SPring-8 Center, RIKEN Harima Institute)

Coherent X-ray diffraction microscopy (CXDM) has appeared as a novel tool for the structural studies of non-crystalline biological particles to be never crystallized. This technique uses intense X-ray beams with good transverse coherence from third-generation synchrotron facilities and X-ray free electron laser (XFEL) sources. The weak interactions of X-rays with electrons enable the penetration into thick samples and the Born approximation in the diffraction process. One of serious problems in the application is radiation damage of samples. Because of the small scattering cross sections of atoms in biological samples, long period exposure causing radiation damage is necessary to collect diffraction data to be analyzed. To overcome radiation damage of biological samples, we recently constructed a system for cryogenic CXDM experiments in $\mathrm{X}$-ray free electron laser and the third generation synchrotron facilities. Here we would like to introduce our system, in particular on two types of diffractometers dedicated for cryogenic CXDM experiments, and preliminary results from cryogenic experiments conducted at BL29XU of SPring- 8 . We also present the method to reconstruct 3D electron densities of sample particles only from diffraction patterns.

\section{SI-03 SACLA in Molecular Imaging \\ Changyong Song (RIKEN SPring-8 Center)}

$\mathrm{X}$-ray crystallography, for more than fifty years, has been the primary methodology to unveil structures of biological molecules at atomic detail. Notwithstanding the remarkable technical advancement, structural investigations of general biological specimens with $\mathrm{x}$-rays have been challenged, often hampered by difficulty in making crystals. Alternatively $x$-ray lens based direct imaging techniques have been developed but the image resolution is limited by the focusing capability of lens itself. The coherent $\mathrm{x}$-ray diffractive imaging technique expects to provide a breakthrough without relying on either a crystal or lens. A series of developments has matured the technique to demonstrate noninvasive $3 \mathrm{D}$ imaging of whole biological cells and organelles at a few tens of nanometer resolution. This imaging technique, further with the advent of bright and short-pulse x-ray lasers, aims to realize near atomic resolution $3 \mathrm{D}$ imaging of single-macromolecules. In this talk, I will introduce our recent progress on biological 3D imaging made by using hard $\mathrm{x}$-rays at SPring-8. Current status and perspective of molecular imaging with SACLA will also be discussed.

\section{$1 \mathrm{SI}-04$}

\section{Visualization of intracellular elements by scanning X-ray fluorescence microscopy}

Mari Shimura ${ }^{1}$, Satoshi Matsuyama ${ }^{2}\left({ }^{1}\right.$ Dept of Intractable Diseases, Res Inst, Nat Centr Global Health \& Med, ${ }^{2}$ Dept of Precision Sci \& Tech Grad Sch of Engineering, Osaka Univ)

Minerals and metals are essential for a healthy body, and the concentrations of these elements have been suggested to change in various cellular conditions and the diseased state. However, the distribution of these intracellular elements was hard to be visualized. We describe the development and use of a scanning X-ray fluorescence microscope (SXFM) system at SPring-8 (Harima, Riken) and an accompanying analytical method (elemental array). We demonstrate that a SXFM can reliably determine the cellular distribution of multiple elements with a high spatial resolution. Visualizing intracellular elements and understanding their kinetics may provide greater insight into cellular kinetics and disease etiology. We also discuss the possible application of X-ray fluorescence microscopy to the fourth-generation light sources, $\mathrm{x}$-ray free electron lasers.

\section{SI-05 X-ray FEL を用いた膜蛋白質構造解析 \\ X-ray FEL and membrane protein crystallography \\ So Iwata (Dep. Cell Biology, Grad. School of Med., Kyoto Univ.)}

Membrane proteins are a supreme example where more effort in structural biology is needed. In spite of their abundance and importance, of the 72,000 protein structures in the Protein Data Bank, only some 250 of these proteins are unique membrane proteins. Many major questions remain unanswered; these include the signal transduction mechanism of $G$ protein coupled receptors (GPCRs), the gating mechanisms of ion channels, and the molecular transport mechanism of transporters. Membrane protein structures are of crucial importance for medicine. Historically, the discovery of new drugs has been led by chemistry and pharmacology. The advent of genomic sciences has opened up the possibility of identification of genetic determinants of different drug responses across a population and the possibility of drug therapy based on the patient's genetic profile. Structural data can inform library design, library screening, selection of targets, optimisation of lead compounds, and engineering of selectivity to improve the overall efficiency of drug discovery. As Chapman and his colleagues showed in a recent paper (Chapman, H.N. et al., Nature 470, 73 (2010)), Xray FEL has a great potential to solve membrane protein structures using multiple micro/nano-crystals. In my talk, I would discuss what we could achieve using SACLA in the field of membrane protein crystallography based on our experience, particularly on the studies performed at the microfocus beamline I24, Diamond light source.

\section{SI-06 Biophysical Imaging with Coherent X-rays}

Keith A Nugent ${ }^{1,2}\left({ }^{1}\right.$ School of Physics, The University of Melbourne, ${ }^{2} A R C$ Centre of Excellence for Coherent X-ray Science)

The Australian Research Council Centre of Excellence for Coherent X-ray Science (CXS) is a major multi-institutional centre devoted to the development of coherent X-ray imaging approaches for application to the biological sciences. In this talk I will outline the work of my centre. In particular, I will discuss the method of Coherent Diffractive Imaging and the contributions we have been making to its development for synchrotron applications ${ }^{1}$. We have paid particular attention to the measurement of imperfect temporal and spatial coherence and the impact it can have on the analysis of the data ${ }^{2}$.

Our work on partial coherence has also enabled us to think of damage processes as a dynamic partial coherence effect. We have recently used these ideas to consider new approaches to the analysis of data from molecules irradiated with light from an X-ray free electron laser ${ }^{3}$.

1.Abbey, B. et al. Keyhole coherent diffractive imaging. Nature Physics 4, 394398 (2008).

2.Whitehead, L.W. et al. Diffractive Imaging Using Partially Coherent X Rays. Physical Review Letters 103, 243902 (2009).

3.Quiney, H.M. \& Nugent, K.A. Biomolecular imaging and electronic damage using X-ray free-electron lasers. Nature Physics 7, 142-146 (2011).

\section{SK-01 マルチコピー・マルチスケール分子動力学シミュレーションプログ ラム開発のためのクラスライブラリ}

A class library for developing multi-copy, multi-scale molecular dynamics simulation programs

Tohru Terada ${ }^{1,2}$, Yasuhiro Matsunaga ${ }^{1,3}$, Kei Moritsugu ${ }^{1}$, Akinori Kidera ${ }^{1,4}$ $\left({ }^{1}\right.$ CSRP, RIKEN, ${ }^{2}$ Grad. Sch. Agri. Life Sci., Univ. Tokyo, ${ }^{3}$ AICS, RIKEN, ${ }^{4}$ Dept. Supramol. Biol., Yokohama City Univ)

One of the important missions of the high performance computing is to clarify the atomistic mechanisms of large motions of proteins accompanied by biologically important phenomena. The time scales of the large motions are typically on the order of microseconds to milliseconds. Performing a conventional all-atom molecular dynamics (MD) simulation on a massively parallel computer is not necessarily an efficient way to study such slow motions, because it is difficult for the normal MD programs to make full use of its computational ability. To cope with this problem, we take a "multi-copy, multi- 
scale" approach based on statistical mechanics. The replica exchange method and the string method are examples of this approach. Here, multiple copies of a molecular system and/or its multiple representations with different resolutions are used to enhance sampling or to perform integration along a path connecting distant points in the conformational space. Since the interactions between the systems are much sparser than those within the systems, the communication cost is much smaller and higher parallel scalability can be achieved. To apply the methods of this approach to various problems of slow dynamics of proteins, we have developed a versatile class library that makes it easy to implement novel multi-copy, multi-scale algorithms. The library is written in an object-oriented language, $\mathrm{C}^{++}$, and is parallelized with OpenMP and MPI. We show how to use this library and present the latest results obtained from its applications.

\section{SK-02 タンパク質透週装置 Sec トランスロコンの分子動カ学シミュレー} ション

Molecular dynamics simulations for the protein secretory pathway

Takaharu Mori ${ }^{1}$, Yuji Sugita ${ }^{1,2,3}\left({ }^{1}\right.$ RIKEN QBiC, ${ }^{2}$ RIKEN AICS, ${ }^{3}$ RIKEN $A S I)$

Advances in massively parallel computing have made possible $1-\mu \mathrm{sec}$ (or longer) simulations of membrane proteins with explicit solvent and membranes, and are beginning to shed considerable light on the biological phenomena in membrane. We have studied molecular mechanisms of the Sec translocon using molecular dynamics (MD) simulations. Sec translocon is a huge membrane protein complex (SecYEG, SecDF, and SecA ATPase) that provides a pathway for secretary proteins to cross membranes, or for membrane proteins to be integrated into membranes. Recently, several crystal structures with different states have been determined for SecYEG, SecDF, and SecA, and it is proposed that each protein undergoes large conformational change during the functional cycle [1,2]. In this study, we performed all-atom MD simulations of the SecY channel to elucidate molecular mechanisms of the conformational transition between the pre-open and closed states. We found that the conformational change is related to the lateral movement of the lipid molecule around the lateral gate region of SecY. We proposed that the intercalation of phospholipids promotes initial entry of the positively-charged signal peptide into the channel $[1,3]$. We will also present our recent works on the MD simulations of SecDF and other membrane proteins.

[1] T. Tsukazaki et al., Nature, 455, 988-992 (2008).

[2] T. Tsukazaki et al., Nature (published online).

[3] T. Mori et al., Biochemistry, 49, 945-950 (2010).

\section{SK-03＼cjkstart創薬における結合自由エネルギー計算法}

Absolute binding free energy calculation for drug development Hideaki Fujitani (RCAST, The University of Tokyo)

Absolute binding free energies of ligands for a pharmaceutical target protein are important to find out drug candidates. If a ligand strongly binds to the protein, it might prevent the protein native activity. Many computation methods have been developed in the past, but it was extraordinary difficult to obtain enough accurate absolute binding free energies for the drug development by computers. We developed the massively parallel computation for the absolute binding free energy (MP-CAFEE) based on the nonequilibrium equality for free energy difference between the two thermodynamic states: bound and unbound states. There are three important requirements for accurate calculations: wellequilibrated binding structure, extensive sampling, and accurate force field. We developed FUJI force field for organic molecules. It has new protein backbone torsion parameters obtained by comparing with high level ab initio calculations for the model molecules of glycine and alanine dipeptides. Our force field shows smaller energy barriers between lower energy structures than the previous force fields like AMBER and OPLS-AA. MP-CAFEE with FUJI force filed gives reliable absolute binding free energies for pharmaceutical target proteins to promote computer aided drug development.

\section{SK-04 スパコンで焙り出すがんのシステム}

\section{Uncovering Systems in Cancer by Supercomputer}

Satoru Miyano (Institute of Medical Science, U. Tokyo)

We present computational methods which boost the challenge for hacking cancer systems by the power of supercomputer. It is impossible to build an individual gene network from one patient sample. However, given many patient samples, it turns possible. We developed a computational method named
NetworkProfiler to exhibit how gene networks vary from patient to patient according to a modulator. The method uses structural equation model for discovering associations between the differences in molecular mechanisms and the diversity of phenotype traits. This method was applied to microarray gene expression profiles of 762 cancer cell lines and unraveled global changes of networks with 20,000 genes of different EMT (epithelial-mesenchymal transition) expression levels. The computation took 90 days using 1024 cores on the supercomputer at Human Genome Center (6000 cores; 75 TFLOPS at peak). Out of 1732 possible regulators of E-cadherin, a cell adhesion molecule that modulates the EMT, we identified 25 candidate regulators, of which about half have been reported in the literature. Some of them were validated as novel genes for EMT including miRNA. We also present computational methods and results using the supercomputer for modeling dynamics in cancer cells from timecourse gene expression profiles that revealed dynamic network changes against anti-cancer drugs and network differences between drug-sensitive and drugresistant cancer cells.

\section{SK-05 統計的学習法を用いた神経スパイクデータからのシナプス結合推定}

\section{A statistical learning method for identifying synaptic} connections from spike train data

Junichiro Yoshimoto ${ }^{1,2}$, Kenji Doya ${ }^{1,2}\left({ }^{1}\right.$ Okinawa Institute of Science and Technology, ${ }^{2}$ Nara Institute of Science and Technology)

Recent progress in neuronal recording technique allows us to record spike events simultaneously from a majority of neurons in a local circuit. Such rich datasets would allow us not only to assess the information coding by neurons but also to uncover the structure of the underlying neural circuit, which allows us to understand the information processing mechanisms. Here, we present a method to identify the synaptic connections from multiple spike train data based on an simple but general neuron model and statistical inference.

The method assumes that spikes are generated from stochastic leaky integrateand-fire neurons connected with multi-exponential postsynaptic current functions. For the model fitting to the data, we develop a Bayesian parameter estimation algorithm with a hierarchical prior that promotes sparseness of the effective parameters. After parameter fitting, three types of synaptic connections (excitatory, inhibitory, or none) are identified based on the maximum amplitude of spike response curves reproduced by the model.

The basic performance of the method was tested in two synthetic benchmarks. The results showed that the proposed Bayesian algorithm could achieve a small variance of estimated model parameters and reproduced the spike response curves well. The method could also distinguish three types of synaptic connectivity with a high classification precision (average AUC score $>99 \%$ in the ROC analysis).

\section{SK-06 ヒトとマウスの筋骨格モーフィングと能張力解析}

\section{Musculoskeletal Morphing from Human to Mouse and Muscle Tension Analysis}

Yosuke Ikegami ${ }^{1}$, Akihiro Yoshimatsu ${ }^{1}$, Ko Ayusawa ${ }^{1}$, Satoshi Oota ${ }^{2}$, Yoshihiko Nakamura ${ }^{1}\left({ }^{1}\right.$ Mechano-Informatics, The University of Tokyo,

${ }^{2}$ BioResource Center, RIKEN)

The laboratory mice are widely used as model animals for studying genetic neurological disorders because of the homology between human and mice. However, the motion analysis technique focusing on the motor function such as muscle tensions or joint torques has not been developed yet. In this talk, we will present a method for generating whole body muscle models of laboratory mice as a first step of the motion analysis. Based on the homology of human and mouse bone geometry, the mapping function between skeletal shapes is designed. With the obtained mapping function, human muscle model is mapped on the mouse skeletal model as the initial mouse muscle model before anatomical confirmation. Mouse musculoskeletal model is partially evaluated by anatomical way, and the estimated muscle length is experimentally analyzed with motion capturing.

\section{SL-01 光合成研究の最前線と課題}

The present of photosynthesis research

Miwa Sugiura (Cell-Free Sci. and Tec. Res. Cent., Ehime Univ.)

The conversion of photon energy to chemical energy by photosynthesis synchronizing with water oxidation is the most important reaction for maintaining all living on the earth, so that the series of photosynthesis reaction occurs quite efficiently. To understanding the relationship between the 